\title{
Development of Urban Planning Process \\ Using Associative Parametric Approach for Optimising Urban Morphology El Adawy, Sherif M.*
}

\author{
(*Correspondent: Eng. Sherif M. El Adawy, Teaching Assistant and MSc Candidate, Architectural \\ Engineering and Environmental Planning Department, Arab Academy for Science and Technology \\ and Maritime Transport, sherif-el-adawy@hotmail.com)
}

\begin{abstract}
:
Regarding the complexity of parameters such as (block size, building heights, streets width, land uses, ... etc.) controlling the aspects of urban planning (UP), traditional tools don't give optimum solutions in reasonable time. These tools are considered static ones, as they depend on coordinate-based systems to link planning elements with each other. Urban planners are now in constant search for new approaches and methodologies to generate multiple parameters of UP projects to overcome timing problem. Among new methodologies is the parametric approach in planning, which offers flexibility to planning variations and explorations of conceptual planning ideas. When disciplines of various science fields merge, and develop, new approaches arise. This paper focuses on merging UP process with an associative parametric approach to address the limitations of existing systems. The associative parametric approach deduces several solutions for the urban planners through utilising computational techniques. This paper reviews the basic concepts of UP regarding its aspects and theories. It illustrates parametric design (PD), its definitions, and techniques. The paper shows how merging the associative parametric approach with the conceptual planning phase of the UP process will lead to the creation of Parameterized Conceptual Planning phase, which can facilitate using several parameters during the UP process. Some examples are also demonstrated to show how applying this new computational technique through its guidelines shown before, hence applied to a case study.
\end{abstract}

Keywords: urban planning process, urban planning; associative parametric approach, parametric design, conceptual planning phase, parametrized conceptual planning phase

ملخص:

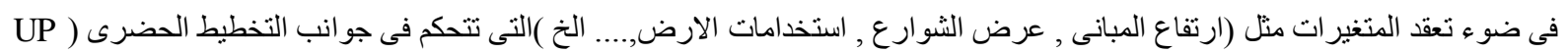

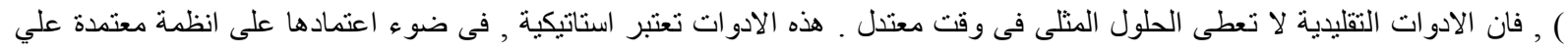

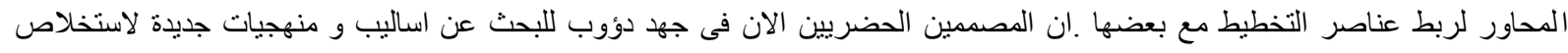

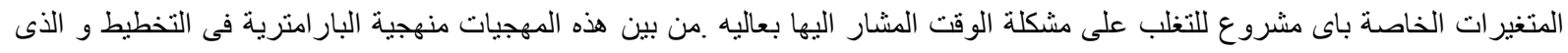

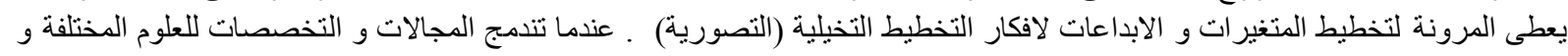

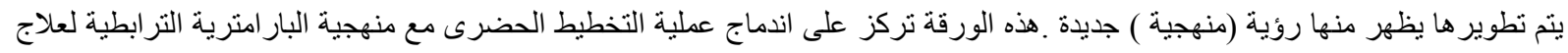

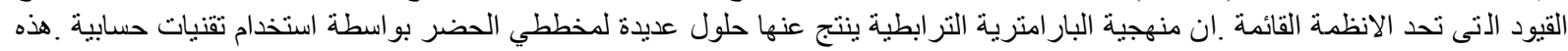

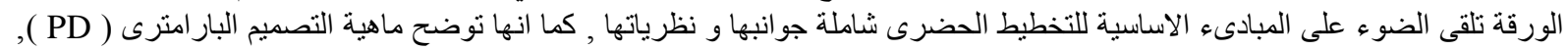

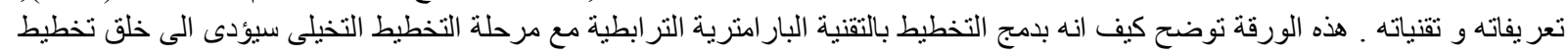

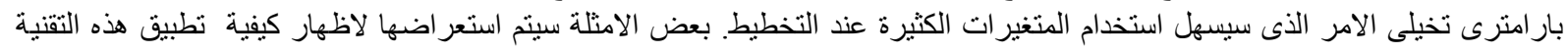

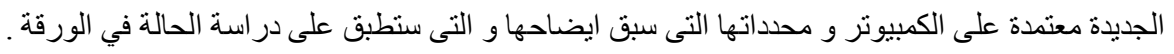




\section{El Adawy, Sherif M. “Development of Urban Planning Process Using Associative Paramet...”}

الكلمات الدالة: علية التخطيط الحضرى التخلى , التخطيط الحضرى التخلى منهجية البار امنرية الترابطية , التصميم البار امترى , مرحلة التخطيط التخيلى ,

\section{INTRODUCTION}

Participatory UP has scenario building, participatory processes have traditionally relied on premeditated planning scenarios, prepared by urban planners. Such planning scenarios can be difficult to modify in the course of participatory processes, as the consequences of changing different parameters cannot easily be evaluated by means of traditional planning tools.

In recent years, new PD tools have opened up new possibilities for a dynamic scenario for UP. By way of a PD approach, different UP parameters can be modified and new urban space scenarios can be rendered three-dimensionally in almost real time. Shortly, this is parametric UP. It opens up completely new possibilities for participatory planning, as anyone can provide input and evaluate the spatial effects instantly in the context of participatory planning processes.

New cities currently experience rapid urban growth, while formal UP is absenting in large parts, resulting in large-scale informal and unplanned development. A parametric UP approach in combination with a more bottom-up planning approach may be the best response to this condition.

This paper aims to investigate the potential for the application of a parametric UP approach to UP in new cities to replace the recent techniques of planning. It will also discuss the solution of problems facing the context of urban development in the new cities.

\section{PROBLEM}

Traditionally, urban plans are developed with rigid and definitive planning systems that do not give optimum solutions in reasonable time. To modify the complexity of UP, it needs a flexible approach to generate multiple solutions.

The computational methods manage and express the increasing complexity of factors that have an effect on the urban morphology method, it will serve planner's needs in the planning process. Consequently, the problem of this paper is declared as following; the traditional tools of dealing with the complexity of parameters aspects of Urban Morphology are no longer able to give the optimum planning solution because they are considered static tools. Therefore, it is essential to consider a collection of rules, parameters, and generators during the planning method that cannot be optimised, unless computational techniques represented in parametric urban models with a multi-criteria approach.

\section{URBAN MORPHOLOGY (UM)}

UM has developed an innovative approach using metric tools to analyse cities' urban fabric. It works with geometric form factors composed of heights, lengths, depths of elements of the city (streets, squares, buildings, blocks...), and pattern form factors to measure additional complex objects like the boundaries of the city. This form issue approach is completed by a graph analysis of street patterns characteristic between the constitution, the configuration and the composition of the connecting layout of the city. The human activities layer is added through an accessible density and diversity analysis based on the theory of information and the Shannon formula (mathematical formula quantifying the mean content in the information of a set of messages). This innovative scientific approach using complementary metric tools is used to analyse cities' urban structure and fabric in addition to related human activities at various scales. Urban metrics provides a structure and an orderable to clarify complex urban problems and helps to find solutions in planning (Salat, 2016).

\subsection{Levels of Urban Morphology}

UM is a discipline focusing on the composition of the urban fabric. The city can be seen as a superposition of six layers. Each one will be studied individually to have a better understanding of the city as a whole (Salat, 2016).

- Level one includes human beings and activities. The interactions between people are the primary issue of organisation of a city. In fact, cities are places of maximisation of exchanges and interactions of all types between people. 
- Level two is the street network. It had been at first created by the real journeys created the most usually by the inhabitants. But today, it is too usually set by engineers almost randomly. Streets networks are a facility however additionally a constraint that forces the inhabitants to follow one way to travel to one purpose and can incite them to use some transport modes.

- Level three is the study of lands. The historic and administrative organisation is a constraint encouraging some varieties of the building. When lands are small, it is difficult to create big towers, and the territory is additional resilient to change. On the contrary, large lands allow for huge buildings and a lot of empty space on the ground.

- Level four is the topography and relief of the site that is an obvious constraint, however additionally something with that architect and urban planners can play.

- Level five is land use and repartition of activities. It affects people flows, housing allocation, and has an economic and social importance

- Level six shows the three dimensions of the city. The solids and the voids determine the air flow and the sun penetration, and thus the dispersion of pollutants and the temperature of the city.

In this paper, will focus on level two and three as guidelines for UM.

\subsection{Methods of Urban Morphology}

Optimum building forms vary according to mixeduse parameters and can have a profound impact on the form of urban spaces. There are two primary approaches to the parametric and quantitative study of the UM in terms of mixed-use aspects (Hsie, 2008).

\subsubsection{Morphometric Method}

This method requires the advanced computation of geometric parameters through the analysis of urban statistical databases. The databases containing information on height, attribute size, shape, size, and arrangement of roughness elements such as buildings and vegetation are required for computation.

\subsubsection{Morphological Method}

The morphological method to survey UM depends primarily on the visual inspection of urban databases or aerial photography.

This paper has a focus on applying the first method only as it deals with the physical parameters of the $\mathrm{UM}$ in order to model lifelike and desirable urban spaces.

\section{ASSOCIATIVE URBANISM APPROACH}

\subsection{Definition}

Associated modelling approach refers to a method where elements are connected in a fixed order, which produces creating a basic for building a new order. Associative planning is based on PD techniques that exploit associative geometry. In PD relationships between objects are explicitly described, establishing interdependencies between the various objects. Variations between objects once generated, it can be easily transformed and manipulated by activating these attributes. Interdependencies between objects and object behaviour can be established under transformation (Salem, Abdelall, Ayad, \& El-Sayad, 2016) (Emmer, 2005).

\subsection{Examples}

Figure 1 and 2 show that DRL Egloo team developed a proposal with an algorithm simulating growth in natural forms and its stages. Space syntax software was then applied in the analysis of the performance of branching systems.

These kinds of coherent, rule-based forms of distribution was initially observed in the flow of viscous fluids spreading across a surface, and the urbanism that emerges from these observations aims to optimise the configuration of the entire site alongside specific local conditions. Between them, self-organized systems control the relation and distribution of varied densities (Leach, 2009).
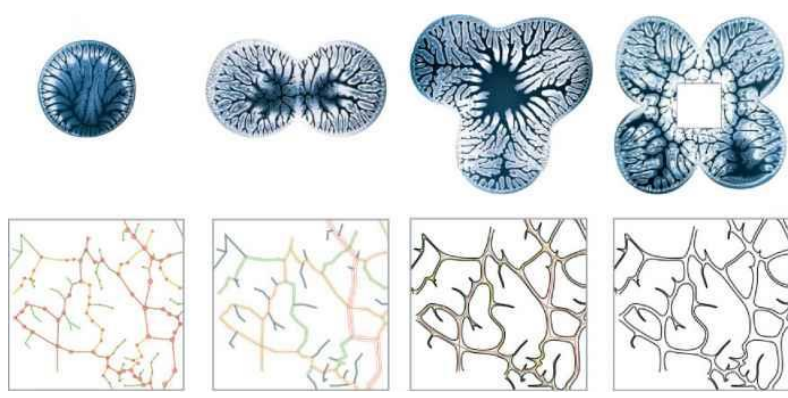

Figure 1, Series of diagrams assessing the neighbourhood qualities of integration and segregation of spaces (Leach, 2009) 


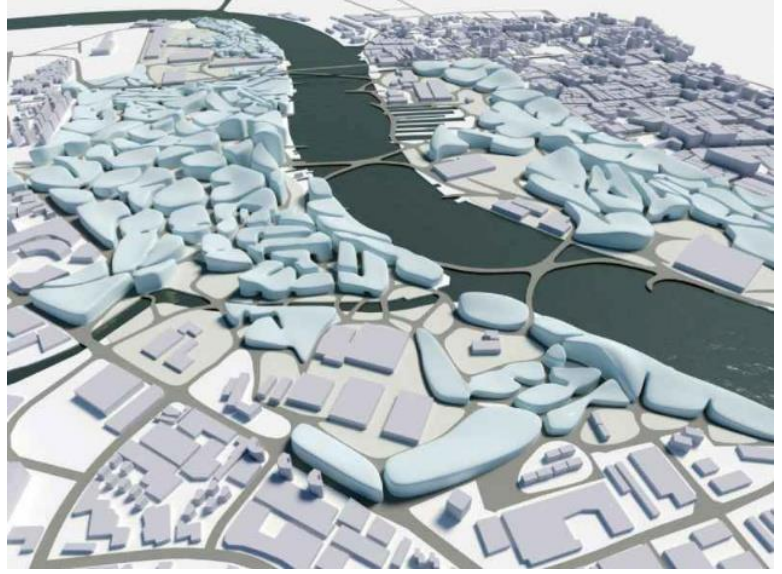

Figure 2, Associative urbanism application by Egloo team in DRL (Leach, 2009)

Zaha Hadid Architects worked on the KartalPendik project required the design of a sub-center on Istanbul's Asian side to reduce pressure on the city's historic core. The site was being reclaimed from industrial estates and was flanked by the small-grain fabric of suburban towns. Therefore, the urban planner should respect the surrounding fabrics, the adjacent context, in particular, the incoming lines of circulation, was taken as an important input for the generation of the urban geometry (Leach, 2009).

Figure 3 shows that associative parametric model called Maya 's hair was applied. This dynamic tool achieved a Parametrically tuned bundling of the incoming paths into larger roads enclosing larger sites such that the resultant lateral path system exhibits the basic properties of Frei Otto 's minimising detour network. The longitudinal direction was imposed via a primary artery with a series of subsidiary roads running parallel. The result is a hybrid of minimising detour network and deformed grid (Leach, 2009).

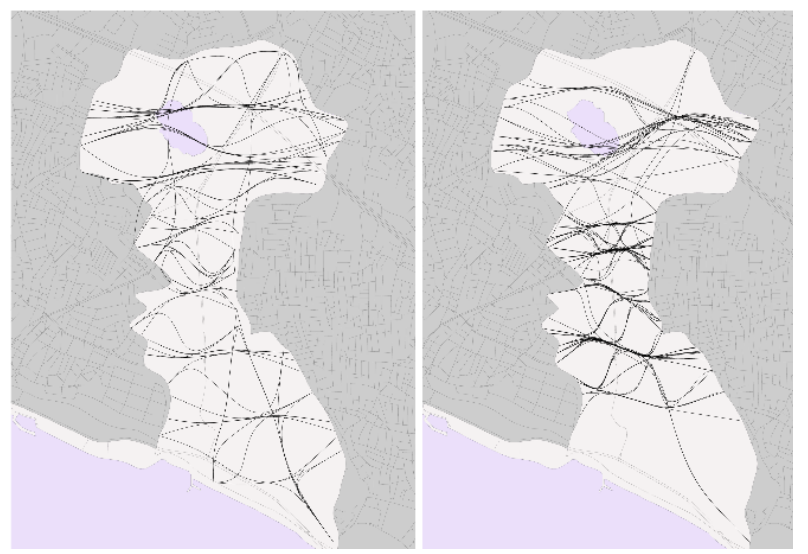

Figure 3, Maya hair-dynamic simulates minimised detour net to generate the paths network to link with the existing fabric (Leach, 2009)
Zaha Hadid Architects worked with two primary fabric typologies, towers and perimeter blocks, each

conceived as a generative component that allows for a wide range of variation. The towers, conceived

as cross towers, were placed on the crossing points to accentuate the path network. The perimeter blocks inversely correlate height with parcel area so that courtyards morph into internal atria as sites get smaller and blocks get taller. And as the final layout is a coherently differentiated cityscape that facilitates navigation through its lawful (rulebased) constitution and its architectural components shown in Figure 4 (Leach, 2009).

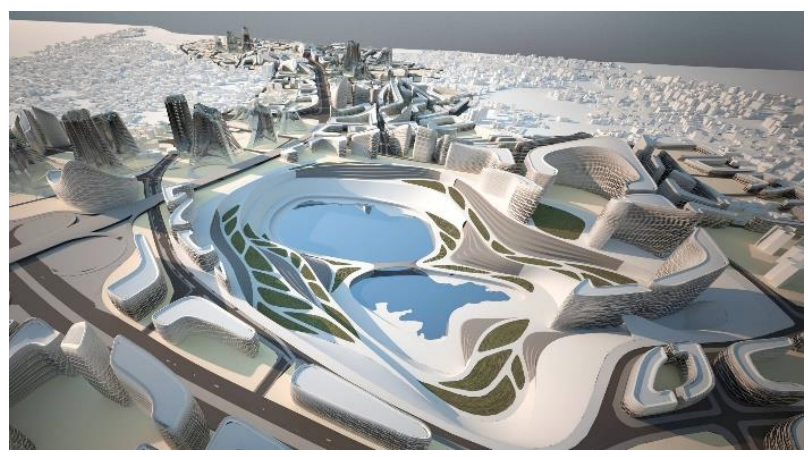

Figure 4 The final layout of the Kartal-Penkik plan which incorporates a vast quarry that becomes the largest item in a system of parks that are spread throughout the urban field. The rhythmic flow of the urban fabric gives a sense of organic cohesion (Leach, 2009)

\section{COMPONENTS OF PARAMETRIC DESIGN}

In a PD, the addition of constraints to geometry to ensure the planning conforms to specified requirements. PD can be defined as a technology that is used for planning with constraints (Law, 2012).

\subsection{Constraints}

Are defined as restrictions and associations that are applied to 2D geometry. There are two types of constraints: geometric and dimensional. Geometric constraints are used to control the relationships of objects in respect to each other. Dimensional constraints are used to control the distance, angle, radius and length values of objects (Law, 2012). 


\subsection{Parameters}

Are used to design, and it change in any design. the product will be different. Parameters are about changing conditions and adaptability to planner needs, that needs to design process instead of objects (Cingolani, 2014).

\subsection{Rules}

Creating rules and dependencies, which then create the design, involved the planners in a higher level of problem framing and definition of the concept of design. It allowed the visualisation and modelling of highly complex forms that may result from nontraditional design data. The planners used their own parametric and rule-based design analyses from the first component and subsequently studied the use and operation of the software, the creation of rules, and parametric and generative design (Schnabel , 2007).

\subsection{Tools}

The direct outcome of such an increasingly persistent requirement for "modern" \& "futuristic" forms, has paved the way for innovative new techniques and tools for today's architect Computer Aided Design (CAD). It deals with the use of computing devices to abet one's odds of better perceiving and visualising a design. It is generally used to create, analyse, modify and finally present the design, due to the accuracy and quality, it is capable of consistently administering (Aprameya, 2016). There are a lot of tools that will get you up and run PD as:

- Generative Components

- Dynamo

- CATIA

- Digital Project

- $\quad$ Autodesk 3DS MAX

- Grasshopper - Rhinoceros

\section{PARAMETRIC URBAN PLANNING PLATFORM}

In this paper, parametric programming will need a $\mathrm{CAD}$ environment as Rhinoceros, and parametric interface as Grasshopper. They will form associative platform or rule to some of the urban planning morphologies (UPM) as streets and size of the blocks to be represented as a parametric engine to generate streets network and blocks division.

Since Grasshopper allows planners to get real-time feedback on parameter changes, one quickly gets an understanding of how variations influence the outcome.
While the parameters are available in the street network components, the planner will create:

- A curve representing the boundary of the project site.

- A set of curves representing existing road to be integrated into the street network

At the same time block components will provide output parameters related to street network:

- The generated street segments as a set of line-like curves.

- A set of closed curves representing urban blocks.

- The corner points of the street blocks grouped correspondingly to the block they belong to.

Street segments can be used to build street profiles. Block boundaries can be used for a further division with the corresponding component to derive a building geometry. The block division component provides a way to subdivide closed curves from the street network into sub-regions. It proceeds by taking a closed base curve, usually the one representing a block. The block is then subdivided into two curves, which together form the shape of the base curve.

\section{APPLICATION}

Figure 6, that has been done by the first author based on previous research to explore all the different interaction between PD and urbanism showing that the planner will consider using the associative and performance-based parametric approaches to reach development of planning in the optimization stage; and to insert a specific plan context in the optimization stage of the plan process. Figure 6 , shows the application of this paper, as to form a definition for Grasshopper, to form Street Network that studies the relations between what we said above (the street network and the block division), Road Types used as parameters to generate streets and its layouts. (Source: the Author).

Parameters used in the definition for Grasshopper will be represented for (A) to (E) and for Rhinoceros represented as a form of curves (R1, $\mathrm{R} 2$, and R3) as shown in Figure 5, which give the types of streets:

- $\quad \mathrm{R} 1$, Spine road

- R2, Major roads

- R3, Minor roads 
(A), controls on R2 and R3. (B), controls on R1 and (C), controls on R3. While (D) and (E), control on the size of the blocks. Figure 7 shows some of the results formed from the definition of Grasshopper as to form Street Network and Road Types (Source: the Author).

As (B) and (C) are a constant value and changing the value of (A), it will control on $\mathrm{R} 2$ and $\mathrm{R} 3$.
While if (A) and (C) are a constant value and changing the value of (B), it will control on R1 only. Finally (A) and (B) are a constant value and changing the value of $(\mathrm{C})$, it will control on R3 only.

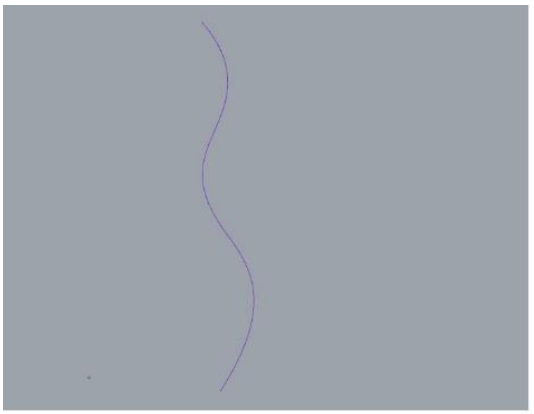

$\mathrm{R} 1$

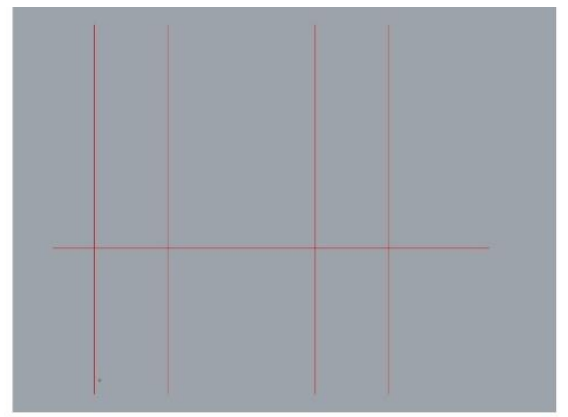

$\mathrm{R} 2$

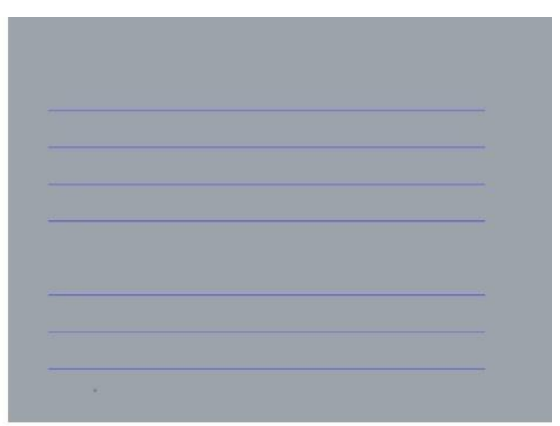

R3

Figure 5, Spine road (R1), Major roads (R2) and Minor roads (R3) (Source: the Author)

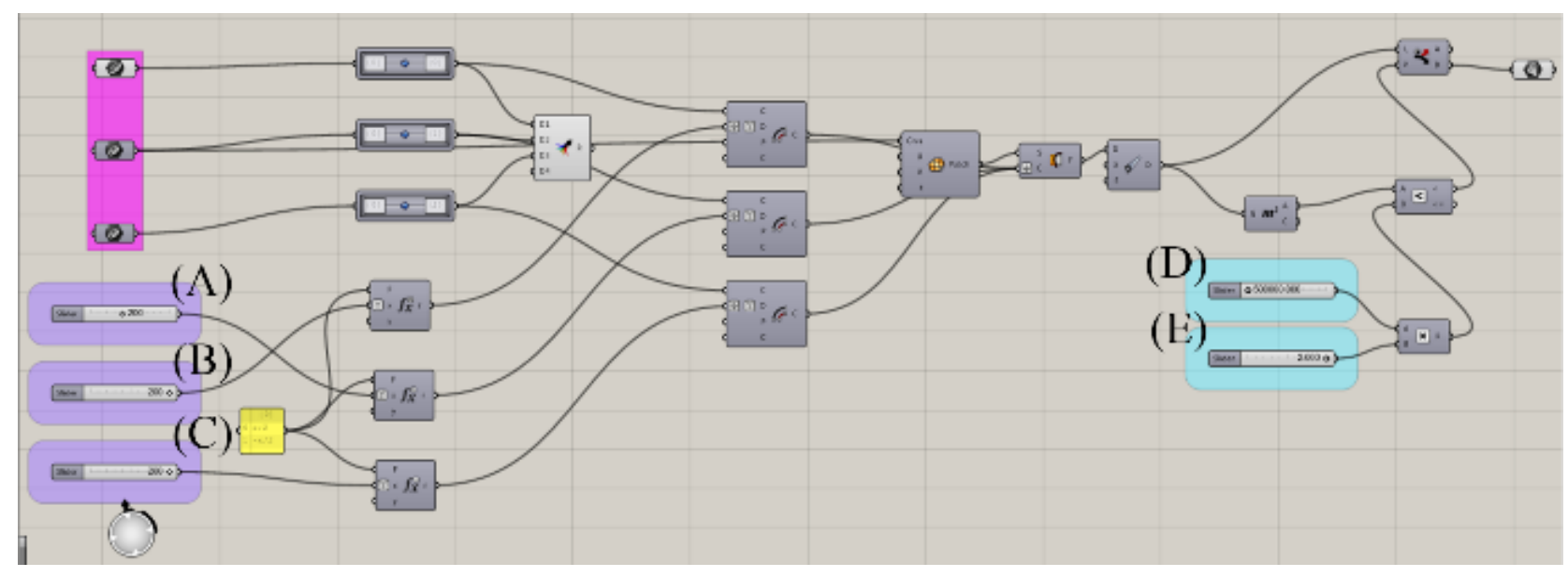

Figure 6, Definition for Grasshopper was formed, to form Street Network and Road Types (Source: the Author) 


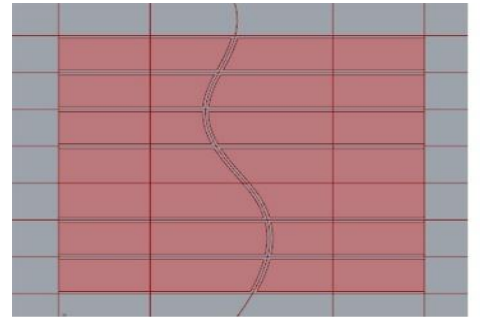

$\mathrm{S} 1$

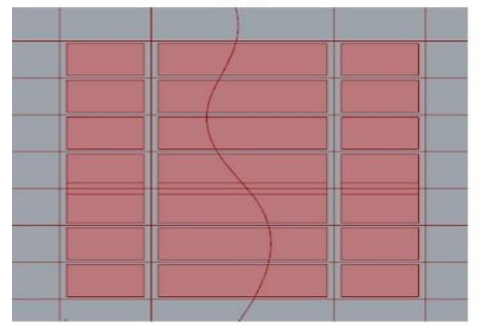

S4

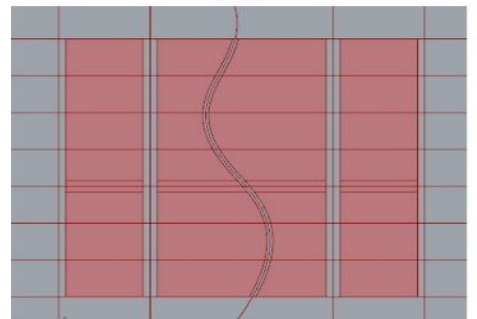

S7

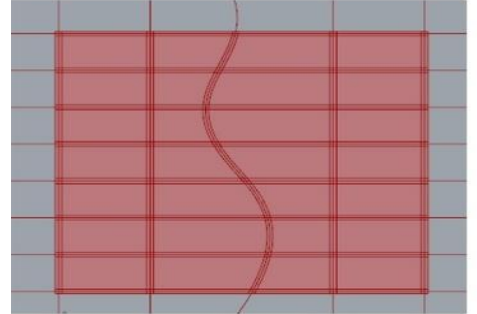

$\mathrm{S} 10$

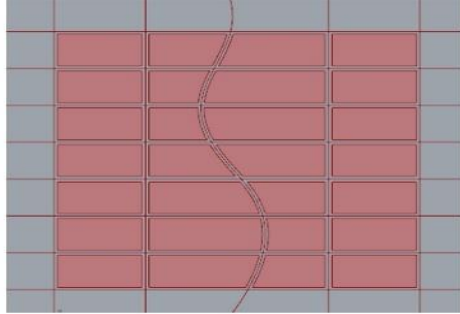

$\mathrm{S} 2$

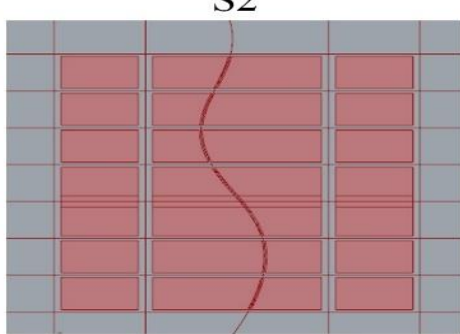

S5

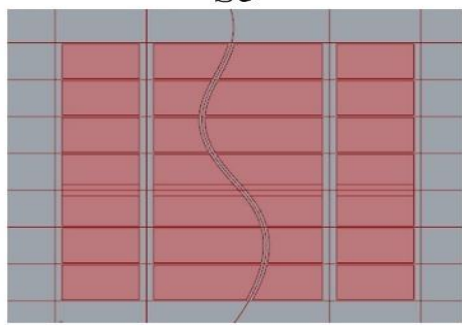

S8

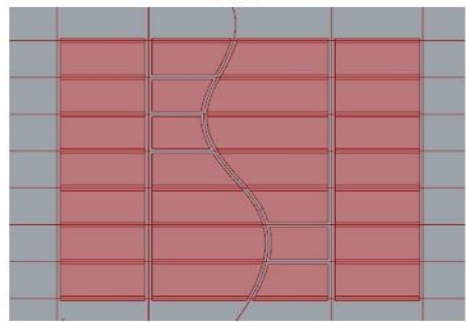

$\mathrm{S} 11$

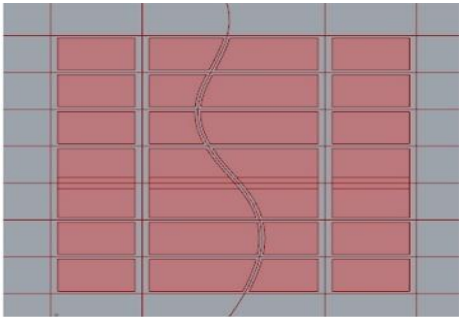

S3

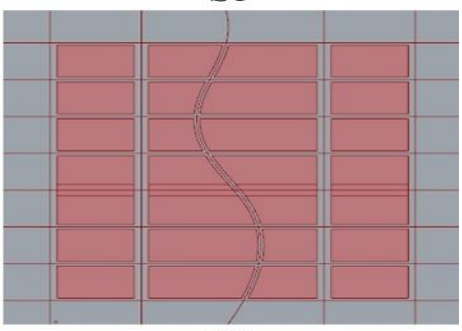

S6

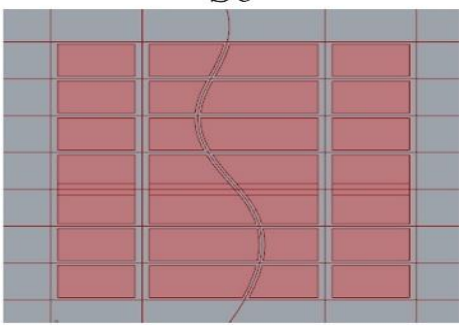

S9

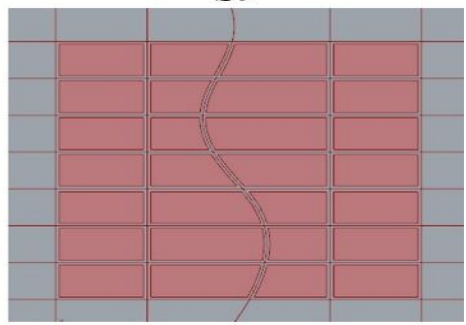

$\mathrm{S} 12$

Figure 7, Some of the results formed from the definition of Grasshopper to form Street Network and Road Types (Source: the Author)

The last process to do is evaluation process generating the design criteria and comparing the design results to choose the best solution from all of them. As for now, the result of the definition formed, S2 is the best solution formed from the platform of UPM because it is the best in the land division and street destitution in a regular nonrandom manner taking in consideration their function refers to Figure 8. This application implemented the advanced computation of geometric parameters mentioned before in the Morphometric method of UM, and how it was achieved by associative modelling approach through the relations between parameters used in the application. This method can be applied on Large-Scale of UP as a city, as it is applied on Small-Scale as a region of a city as mentioned in this application.

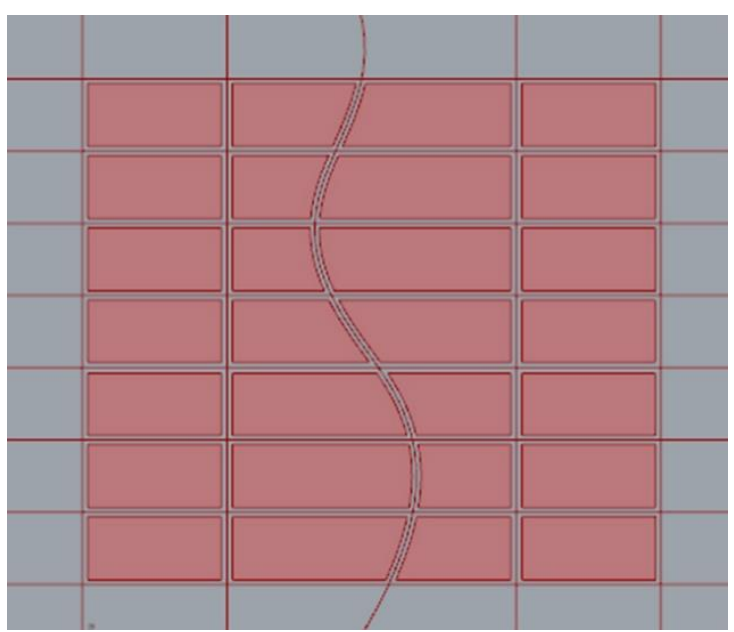

Figure 8, S2 is the best solution (Source: the Author) 


\section{CONCLUSION AND FUTURE WORK}

The parametric method brings remarkable result in the planning process. The results are hardly obtained by conventional methods. The method accommodates a combination of form parameters with various assessment tools or algorithms for UPM. The algorithms are useful for various analysis, e.g. land use, climate, structure, and others. The quality of planning of UPM is enhanced because it is based on scientific analysis. The modelling analysis introduced by this method enabled the planner to monitor changes during the planning process, which in turn, enhance the understanding and knowledge of design.

In our future work, the development of UP will concentrate on the development of evaluation and optimisation methods for associative parametric UM generated by the components. It would be useful to incorporate land use information, e.g. to create more meaningful networks by taking into consideration the using of building with its height variations.

\section{References}

Aprameya. (2016, May 20). 10 Parametric Plugins Every Architect Should Know. Retrieved from arch2o: http://www.arch2o.com/10parametric-plugins-every-architectshould-know/

Cingolani, F. (2014, March 21). Parametric VS Computational Design.

Emmer, M. (2005). Mathland: The Role of Mathematics in Virtual Architecture. Nexus Network Journal, 73-88.

Hsie, T.-S. (2008). Achieving Urban Sustainability through Urban Morphology. Conference on Passive and Low Energy Architecture. Dublin,: PLEA.

Law, M. (2012, January 25). Understanding Parametric Constraints.

Leach, N. (2009). Architectural Design-Digital Cities. Wiley.

Salat, S. (2016, May 20). Sustainable Arabic Urban Design. Retrieved from Urban Morphology Complex Systems Institute: http://www.urbanmorphologyinstitute.org/ resources/sustainable-arabic-urban-design/

Salem, N., Abdelall, M., Ayad, H., \& El-Sayad, Z. (2016). The Implementations of Parametric Design Tool in The Urban Environment to Achieve Quality of Life. Proceedings of 2016 2nd International Conference on Architecture, Structure and Civil Engineering, 167-173.

Schnabel , M. A. (2007). Parametric Designing in Architecture . Springer, 238-250. 